\title{
Factors Associated with Postpartum Family Planning in a Cohort of Kelantanese Women with Previous Caesarean Delivery in Malaysia
}

\author{
Hafizah I ${ }^{a}$, Tengku Alina $\mathrm{TI}^{\mathrm{a}}$, Suhaily $\mathrm{MH}^{\mathrm{a}}$, Zaharah $\mathrm{S}^{\mathrm{b}}$ \\ aDepartment of Community Medicine, Universiti Sains Malaysia \\ bWomen's Health Development Unit, Universiti Sains Malaysia
}

\section{ABSTRACT}

Introduction: This community-based, cross-sectional study aimed to identify the factors associated with postpartum family planning use among a cohort of women with recent caesarean delivery in a state with the lowest contraceptive use in Malaysia. Materials and Methods: A total of 281 women aged between 18-49 years old who had caesarean delivery in government tertiary centres in Kelantan from January until April 2017 were enrolled in this study. The study was conducted from January until April 2018. Women were selected through stratified random sampling with probability proportional to size. Data were collected through a validated structured questionnaire. The main outcome was binary (postpartum family planning use or non-use). The factors included socio-demographic details, reproductive history, previous contraceptive use, contraceptive health education received, knowledge, and social support. Simple and multiple logistic regression were conducted to identify significant determinants of postpartum family planning use. Results: The factors associated with postpartum family planning use included a secondary and below education level of women $(\mathrm{AOR}=2.37,95 \% \mathrm{Cl}(1.05,5.34))$, previous contraceptive use $(\mathrm{AOR}=9.82,95 \% \mathrm{Cl}(4.81,20.06))$, individual health education $(\mathrm{AOR}=4.19,95 \% \mathrm{Cl}(1.23,14.30))$, higher knowledge score $(\mathrm{AOR}=1.12,95 \% \mathrm{Cl}$ $(1.03,1.23))$, and higher social support score $(A O R=1.09,95 \% \mathrm{Cl}(1.03,1.16))$. Conclusions: here remains a need to enhance personalised contraceptive counselling in the primary care setting as well as to promote longer acting reversible contraceptive methods.

KEYWORDS: Postpartum, Contraception, Caesarean, Knowledge, Social support

\section{INTRODUCTION}

Postpartum family planning (PPFP), defined as the use of contraceptives within the first year after childbirth to prevent unintended and closely spaced pregnancies ${ }^{1}$, was deemed significant in achieving the United Nations Sustainable Development Goals

Corresponding Author:

Dr. Tengku Alina Tengku Ismail

Department of Community Medicine, School of Medical Sciences, Health Campus, Universiti Sains Malaysia, 16150 Kubang Kerian, Kelantan, Malaysia Tel No : +60199389558

Email : dralina@usm.my related good health and wellbeing as well as gender equality. ${ }^{2}$ A previous study indicated that despite $65 \%$ of postpartum women preferring to space or limit childbirth, they were not using effective contraceptives within the first year following delivery. ${ }^{3}$ Previous data on the contraceptive prevalence rate among women in the general Malaysian population indicated stagnation for the past 35 years with only approximately $30 \%$ of women were using contraceptives ${ }^{4}$, with limited data available on postpartum family planning use. Women in Malaysia was found to practice traditional methods 
using herbal preparations for contraception ${ }^{5}$ which may include use of Malay folk methods such as jamu. ${ }^{6}$

Increasing trend in caesarean rates had been observed both globally and throughout Malaysia. ${ }^{7,8}$ Overall, one-fifth of women with previous caesarean delivery had closely-spaced pregnancy 9, 10 despite the recommendation that women should wait at least 24 months before embarking on a subsequent pregnancy. ${ }^{11}$ Closely-spaced pregnancies among those with previous caesarean delivery placed them at higher risk of complications such as uterine rupture ${ }^{12}$ and adverse perinatal as well as infant outcomes including preterm birth, low birth weight, and small for gestational age. ${ }^{9,12,13}$ These complications were attributed to maternal depletion syndrome, whereby women had not fully recovered from the preceding pregnancy to support a subsequent pregnancy. ${ }^{13}$ The use of the lactational amenorrhea method (LAM) which was exclusively for postpartum women-was also compromised, as research has indicated that caesarean deliveries negatively affect breastfeeding. ${ }^{14}$ Realising the importance between the rising trend in caesarean delivery and the need for PPFP use, this study aimed to determine the factors associated with PPFP use in a cohort of Malaysian women with recent caesarean delivery.

\section{MATERIALS AND METHODS}

\section{Study design and participants}

This community-based, cross-sectional study involved primary data collection from January until April 2018 among women who had caesarean delivery in government tertiary centres in Kelantan. Kelantan is in the north-eastern portion of Peninsular Malaysia. Notably, this area exhibits the lowest contraceptive prevalence in the country. ${ }^{15}$

There are four government tertiary centres in Kelantan; Hospital Raja Perempuan Zainab II (HRPZ II), Hospital Universiti Sains Malaysia (HUSM), Hospital
Kuala Krai (HKK), and Hospital Tanah Merah (HTM). Participants were enrolled from all these tertiary centres.

Sample size was calculated using Power and Sample Size (PS) Software version 3.0 (two proportion formula). ${ }^{16}$ The parameters and values used to calculate the sample size; $\mathrm{p}_{0}=$ probability of the factor in non-PPFP user ${ }^{23}, \mathrm{p}_{1}=$ probability of the factor in PPFP user, $\mathrm{m}=$ ratio between non-PPFP and PPFP user $=0.5^{23}$. The required sample size was 280 after considering $20 \%$ probability of missing data. A sampling frame was formed from the list of women who had caesarean delivery from January until April 2017 at each respective centre. Participants were selected through stratified random sampling with probability proportional to size with the strata being the tertiary centre where the participants delivered. All women in the extended postpartum period; within 12 months after delivery, that underwent caesarean delivery in government tertiary centres in Kelantan from January until March 2017 were eligible for this study. Those who had caesarean hysterectomy during caesarean delivery, were illiterate, diagnosed with a psychiatric illness, or divorced were excluded. Those who resided outside of Kelantan and only returned to Kelantan to deliver were also excluded.

The contact information of women who had caesarean delivery was obtained from each respective centre. Selected women were contacted and had the study protocol explained to them.Once they verbally agreed to participate in the study, a written informed consent form was delivered to their postal address. Subsequent interviewer-guided questionnaires were completed through a 20-minute telephone interview conducted by a single researcher to reduce inter-rater and information bias.

This study was conducted in accordance with ethical principles and received ethical approval from the Ministry of Health Malaysia (NMRR-17-2674-38640) and Universiti Sains Malaysia (USM/JEPeM/17110592). 
The primary outcome was dichotomous (PPFP use and non-use). PPFP use was defined as occurring among those who initiated and continued the use of modern contraceptives within the first 12 months following childbirth. These include the use of an intrauterine device, condoms/spermicides, diaphragm/cervical cap, progestogen-only methods, combined hormonal methods, and sterilisation. ${ }^{3,17}$

According to the social ecological model, multiple influences served as determinants of health behaviour. ${ }^{18}$ Thus, this study included intrapersonal (individual), interpersonal, and organisational determinants. Socio-demographics (age, education level, employment status, and household income), reproductive history (age and duration of marriage, gravidity, parity, history of short birth spacing, mistimed, unwanted or induced abortion), previous contraceptive use, and knowledge operating at the individual level, social support at the interpersonal level, and contraceptive health education at organisational level.

Health education included any combination of learning experience designed to help individuals or communities improve their health by increasing knowledge or influencing attitudes. ${ }^{19}$ Hence, contraceptive health education referred to selfreporting of having received health education on contraception during the antenatal period, during/ immediately after delivery, or during the postpartum period either individually or in a group (with or without spouse involvement).

\section{Research tool and data collection}

This study utilised primary data collected through an interviewer-guided validated questionnaire in the Malay language ${ }^{20}$. The questionnaire consisted of five parts: The first part encompassed socio-demographic details (age and education level of women and their husbands, employment status, and household income). The second part involved reproductive history (age and duration of marriage, gravidity, parity, history of short birth spacing, mistimed, unwanted or induced abortion, and previous and current contraceptive use). The third part covered contraceptive health education concerning the timing of health education, and whether it was conducted individually or involved the spouse. The fourth part involved contraceptive and reproductive health knowledge. The final part assessed the social support received by women. The Cronbach's alpha coefficient for contraceptive knowledge was 0.78 , for reproductive health knowledge was 0.71 and for social support was 0.86 . The Cronbach's alpha coefficient was more than 0.70 for these scales with adequate reliability which were applicable to the last two parts of the questionnaire used.

There was a total of 20 questions regarding knowledge; two open-ended questions on women's awareness of modern contraceptive methods, 11 items on contraceptive knowledge and 7 items on reproductive health knowledge. Contraceptive knowledge questions related to the mode of actions and administration of various contraceptives. Items assessing reproductive health knowledge comprised of questions on the menstrual cycle, reproductive system physiology and pathology, including sexually transmitted infections and HIV. The options for these questions included "true", "false", or "don't know". The total score was 18; 1 point was awarded for the correct answer, while no score was provided for wrong or "don't know" answers. Knowledge was further categorised into "good" (for those that scored above the $75^{\text {th }}$ percentile), "moderate" (for those that scored between the $50^{\text {th }}$ and $75^{\text {th }}$ percentile), or "poor" (for those that scored below the $50^{\text {th }}$ percentile).

The final part (social support) contained seven items 
that assessed perceived social support from the husband (three items) and peers (four items). A fivepoint Likert scale was used; "1: Strongly disagree", “2: Disagree", “3: Neutral”, “4: Agree”, and "5: Strongly agree". The possible score ranged from 7 to 35. The total score for each respondent's perceived social support was calculated and categorised into "poor" (lower than the $50^{\text {th }}$ percentile), "moderate" $\left(50^{\text {th }}\right.$ to $75^{\text {th }}$ percentile $)$ and "good" (greater than the $75^{\text {th }}$ percentile).

\section{Data analysis}

Data were entered and analysed using IBM SPSS version 24 software. ${ }^{21}$ Simple and multiple logistic regression analysis was performed to determine the factors associated with PPFP use. Simple logistic regression was used to select the preliminary variables associated with PPFP use. Variables with $p$ value $<0.25$ or that were clinically relevant and important were included in the multiple logistic regression analysis. The preliminary main effect model was obtained after comparing models using the backward likelihood ratio (LR) and forward LR methods. Model fitness was tested using the Hosmer and Lemeshow goodness of fit test, a classification table, and the receiver operating characteristic (ROC) curve. The significance level was set at 0.05 .

\section{RESULTS}

\section{Study participants}

A total of 281 women between 12-15 months postcaesarean delivery from government tertiary centres were included (Table 1). The mean (SD) duration postpartum was $12.4(0.64)$ months. Nine women were already pregnant at the time of interview; one was due to method failure (pregnant while using an intrauterine device), one pregnancy was due to infrequent condom use (alternating between condom and the withdrawal method), three were using the withdrawal method, and one thought that she was protected through breastfeeding, while three were not using any contraceptive methods.

The youngest respondent was 21 years old, and the maximum respondent age was 46 years old. Out of 281 women, 87 (31\%) were of advanced maternal age (35 years old and above). ${ }^{22}$ Most of the respondents received tertiary education (74\%) and were employed (61\%). The husbands of respondents were between 20 and 60 years old. The youngest age of marriage was 16 years old, and the latest was 35 years old. Of the 281 women, 16 (5.7\%) had 5 or more living children (i.e. grand multiparous). Although most of the respondents had tertiary level education (74\%) and were employed (61\%) with more than $90 \%$ received contraceptive health education, only $19.2 \%$ obtained a good knowledge score. Less than one-quarter $(22.4 \%)$ perceived receiving good social support from husbands and peers. Nearly twothirds $(65.5 \%)$ of respondents were using postpartum family planning, with contraceptive pills and condoms being the two most popular methods. Similarly, contraceptive pills and condoms were the main methods used among those who had experience in using a contraceptive method. Among those of advanced maternal age, 58 (66.7\%) were using PPFP. Approximately $20 \%$ of respondents who were grand multiparous were not using any contraceptives. A total of 20 out of 50 respondents (40\%) who were still breastfeeding their children were not using contraceptives. Less than half of respondents (44\%) were aware of vasectomy as method of contraception. Most women received contraceptive health during their in-patient hospital stay when admitted for labour and delivery.

\section{Trend of postpartum family planning initiation}

The median (IQR) for time of initiation of postpartum family planning was 1.0 (3.0) month. Approximately $30 \%$ initiated PPFP use within the first month, 53\% initiated it within three months, and 61\% initiated it within 6 months of delivery (Figure 1). 
Table I: Description of women involved in the study $(\mathrm{n}=281)$

\begin{tabular}{|c|c|c|}
\hline Variable & Mean (SD) & $\mathrm{n}(\%)$ \\
\hline $\begin{array}{c}\text { Tertiary centre } \\
\text { HRPZ II } \\
\text { HUSM } \\
\text { HTM } \\
\text { HKK }\end{array}$ & & $\begin{array}{l}136(48.4) \\
93(33.1) \\
28(10.0) \\
24 \quad(8.5)\end{array}$ \\
\hline Socio-demographic & & \\
\hline Age of women (year) & $32.12(5.09)$ & \\
\hline $\begin{array}{l}\text { Education level of women } \\
\text { Primary } \\
\text { Secondary } \\
\text { Tertiary }\end{array}$ & & $\begin{array}{cc}2 & (0.7) \\
71(25.3) \\
208(74.0)\end{array}$ \\
\hline $\begin{array}{l}\text { Employment status } \\
\text { Unemployed } \\
\text { Employed }\end{array}$ & & $\begin{array}{l}109(38.8) \\
172(61.2)\end{array}$ \\
\hline Age of husband (year) & $34.25(6.41)$ & \\
\hline $\begin{array}{l}\text { Education level of husband } \\
\text { No formal education } \\
\text { Primary } \\
\text { Secondary } \\
\text { Tertiary }\end{array}$ & & $\begin{array}{r}(0.4) \\
(0.7) \\
117(41.6) \\
161(57.3)\end{array}$ \\
\hline Household income (RM) & $3000.00(4500.00)^{a}$ & \\
\hline Reproductive history & & \\
\hline Age of marriage (years) & $25.23(3.48)$ & \\
\hline Duration of marriage (years) & $5.00(6.00)^{\mathrm{a}}$ & \\
\hline Gravidity & $2.00(2.00)^{\mathrm{a}}$ & \\
\hline Parity & $2.00(2.00)^{\mathrm{a}}$ & \\
\hline Number of living children (boys) & $1.00(2.00)^{\mathrm{a}}$ & \\
\hline Number of living children (girls) & $1.00(2.00)^{\mathrm{a}}$ & \\
\hline $\begin{array}{l}\text { Birth spacing less than } 2 \text { years } \\
\text { Yes } \\
\text { No }\end{array}$ & & $\begin{array}{c}50(17.8) \\
231(82.2)\end{array}$ \\
\hline $\begin{array}{l}\text { History of mistimed pregnancy } \\
\text { Yes } \\
\text { No }\end{array}$ & & $\begin{array}{c}60(21.4) \\
221(78.6)\end{array}$ \\
\hline $\begin{array}{l}\text { History of unwanted pregnancy } \\
\text { Yes } \\
\text { No }\end{array}$ & & $\begin{array}{c}35(12.5) \\
246(87.5)\end{array}$ \\
\hline $\begin{array}{l}\text { History of induced abortion } \\
\text { Yes } \\
\text { No }\end{array}$ & & $\begin{array}{cc}12 & (4.3) \\
269 & (95.7)\end{array}$ \\
\hline $\begin{array}{l}\text { Previous contraceptive use } \\
\text { Yes } \\
\text { No }\end{array}$ & & $\begin{array}{c}206(73.3) \\
75(26.7)\end{array}$ \\
\hline Previous contraceptive methods used ${ }^{\mathrm{b}}$ & & $206(73.3)$ \\
\hline $\begin{array}{l}\text { Contraceptive pills } \\
\text { Male condoms } \\
\text { Injections } \\
\text { Intrauterine devices } \\
\text { Implants }\end{array}$ & & $\begin{array}{l}115(55.8) \\
71(34.5) \\
61(29.6) \\
12(5.8) \\
6(2.9)\end{array}$ \\
\hline Contraceptive health education & & \\
\hline $\begin{array}{l}\text { Received health education } \\
\text { Yes } \\
\text { No }\end{array}$ & & $\begin{array}{cc}256 & (91.1) \\
25 & (8.9)\end{array}$ \\
\hline $\begin{array}{l}\text { Antenatal health education } \\
\text { Yes } \\
\text { No }\end{array}$ & & $\begin{array}{c}97(34.5) \\
184(65.5)\end{array}$ \\
\hline
\end{tabular}




\begin{tabular}{|c|c|c|}
\hline Variable & Mean (SD) & $\mathrm{n}(\%)$ \\
\hline $\begin{array}{l}\text { In-patient health education } \\
\text { Yes } \\
\text { No }\end{array}$ & & $\begin{array}{l}171(60.9) \\
110(39.1)\end{array}$ \\
\hline $\begin{array}{l}\text { Postnatal health education } \\
\text { Yes } \\
\text { No }\end{array}$ & & $\begin{array}{c}78(27.8) \\
203(72.2)\end{array}$ \\
\hline $\begin{array}{l}\text { Child immunization visit health education } \\
\text { Yes } \\
\text { No }\end{array}$ & & $\begin{array}{l}146(52.0) \\
135(48.0)\end{array}$ \\
\hline $\begin{array}{l}\text { Individual health education }{ }^{c} \\
\text { Yes } \\
\text { No }\end{array}$ & & $\begin{array}{cc}238 & (84.7) \\
18 & (6.4)\end{array}$ \\
\hline $\begin{array}{l}\text { Spousal involvement during health educa } \\
\text { Yes } \\
\text { No }\end{array}$ & & $\begin{array}{c}58(20.6) \\
198(70.5)\end{array}$ \\
\hline Aware of modern contraceptive methods ${ }^{d}$ & & $248(88.3)$ \\
\hline $\begin{array}{l}\text { Contraceptive pills } \\
\text { Male condoms } \\
\text { Injections } \\
\text { Intrauterine devices } \\
\text { Implants } \\
\text { Bilateral tubal ligation } \\
\text { Vasectomy }\end{array}$ & & $\begin{array}{l}241(97.2) \\
232(93.5) \\
245(98.8) \\
228(91.9) \\
231(93.1) \\
195(78.6) \\
109(44.0)\end{array}$ \\
\hline $\begin{array}{l}\text { Knowledge score } \\
\text { Good (16-18) } \\
\text { Moderate }(12-15) \\
\text { Poor }(0-11)\end{array}$ & $12.00(6.00)^{\mathrm{a}}$ & $\begin{array}{l}54(19.2) \\
105(37.4) \\
122(43.4)\end{array}$ \\
\hline $\begin{array}{l}\text { Social support score } \\
\text { Good (33-35) } \\
\text { Moderate }(28-32) \\
\text { Poor }(0-27)\end{array}$ & $28.00(9.00)^{\mathrm{a}}$ & $\begin{array}{l}63(22.4) \\
78(27.8) \\
140(49.8)\end{array}$ \\
\hline Current methods used & & \\
\hline Postpartum family planning use & & $184(65.5)$ \\
\hline $\begin{array}{l}\text { Contraceptive pills } \\
\text { Male condoms } \\
\text { Bilateral tubal ligations } \\
\text { Injections } \\
\text { Intrauterine devices } \\
\text { Implants } \\
\text { Mixed (pills+condoms) }\end{array}$ & & $\begin{aligned} 55 & (29.9) \\
45 & (24.5) \\
36 & (19.6) \\
31 & (16.8) \\
7 & (3.8) \\
5 & (2.7) \\
5 & (2.7)\end{aligned}$ \\
\hline Non-users & & $97(34.5)$ \\
\hline Traditional methods & & $67(69.1)$ \\
\hline $\begin{array}{l}\text { Abstinence } \\
\text { Breastfeeding } \\
\text { Calendar method } \\
\text { Withdrawal } \\
\text { Herbal preparations } \\
\text { Jamu } \\
\text { Ubat tabib }\end{array}$ & & $\begin{array}{c}10(10.3) \\
28(28.9) \\
16(16.5) \\
33(34.0) \\
12(12.4) \\
10(10.3) \\
2(2.1)\end{array}$ \\
\hline Not using any method & & $30(30.9)$ \\
\hline
\end{tabular}

a Median (IQR)

b For those that used contraceptives, the total may be more than 100\% (women may have experience with more than one method)

c For those that received health education

d For those that were aware of modern contraceptive methods, total may be more than $100 \%$

e Total percentage may be more than $69.1 \%$ (women may use a combination of traditional methods)

characteristics (ROC) curve was $82.7 \%$ 
Table II: Simple logistic regression of factors associated with PPFP use $(n=281)$

\begin{tabular}{|c|c|c|c|c|c|c|c|}
\hline \multirow{3}{*}{ Factors } & \multicolumn{4}{|c|}{ PPFP use } & \multirow{3}{*}{$\begin{array}{l}\text { Crude odds ratio } \\
\qquad(95 \% \mathrm{Cl})\end{array}$} & \multirow{3}{*}{$\begin{array}{l}\text { Wald } \\
\text { statistics } \\
\text { (df) }\end{array}$} & \multirow{3}{*}{$p$-value } \\
\hline & \multicolumn{2}{|c|}{ No $(n=97)$} & \multicolumn{2}{|c|}{ Yes $(n=184)$} & & & \\
\hline & Mean (SD) & n (\%) & Mean (SD) & n (\%) & & & \\
\hline \multicolumn{8}{|l|}{ Socio-demographic } \\
\hline Age of women (year) & $\begin{array}{r}31.85 \\
(4.58)\end{array}$ & & $\begin{array}{r}32.27 \\
(5.35)\end{array}$ & & $1.02(0.97,1.07)$ & $0.45(1)$ & 0.504 \\
\hline \multicolumn{8}{|l|}{$\begin{array}{l}\text { Education level of } \\
\text { women** }^{* *}\end{array}$} \\
\hline $\begin{array}{l}\text { Tertiary } \\
\text { Secondary and } \\
\text { below }\end{array}$ & & $\begin{array}{c}7938.0) \\
18(24.7)\end{array}$ & & $\begin{array}{r}129(62.0) \\
55(75.3)\end{array}$ & $1.87(1.03,3.41)$ & $4.17(1)$ & 0.041 \\
\hline $\begin{array}{c}\text { Employment status* } \\
\text { Housewife } \\
\text { Working }\end{array}$ & & $\begin{array}{l}44(40.4) \\
52(30.6)\end{array}$ & & $\begin{array}{r}65(59.6) \\
118(69.4)\end{array}$ & $1.52(0.92,2.51)$ & $2.68(1)$ & 0.102 \\
\hline $\begin{array}{l}\text { Age of husband } \\
(\text { year })^{*}\end{array}$ & $\begin{array}{r}33.49 \\
(5.54)\end{array}$ & & $\begin{array}{r}34.65 \\
(6.80)\end{array}$ & & $1.03(0.99,1.07)$ & $2.06(1)$ & 0.151 \\
\hline \multicolumn{8}{|l|}{$\begin{array}{l}\text { Education level of } \\
\text { husband }\end{array}$} \\
\hline below Tertiary & & $53(32.9)$ & & $108(67.1)$ & $1.18(0.72,1.94)$ & $0.43(1)$ & 0.514 \\
\hline $\begin{array}{l}\text { Household income } \\
(\mathrm{RM})^{*}\end{array}$ & $\begin{array}{r}3701.89 \\
(2680.73)\end{array}$ & & $\begin{array}{r}4454.66 \\
(3388.18)\end{array}$ & & $1.01(1.00,1.02)$ & $3.35(1)$ & 0.067 \\
\hline \multicolumn{8}{|l|}{ Reproductive history } \\
\hline $\begin{array}{l}\text { Age of marriage } \\
\text { (year) }{ }^{*}\end{array}$ & $\begin{array}{r}25.73 \\
(3.16)\end{array}$ & & $\begin{array}{r}24.96 \\
(3.61)\end{array}$ & & $0.94(0.87,1.01)$ & $3.09(1)$ & 0.079 \\
\hline $\begin{array}{l}\text { Duration of marriage } \\
(\text { year })^{*}\end{array}$ & $\begin{array}{r}6.11 \\
(4.59)\end{array}$ & & $7.31(5.42)$ & & $1.05(0.99,1.10)$ & $3.35(1)$ & 0.067 \\
\hline Gravidity* & $\begin{array}{r}2.25 \\
(1.33)\end{array}$ & & $2.59(1.65)$ & & $1.17(0.98,1.38)$ & $3.10(1)$ & 0.078 \\
\hline Parity** & $\begin{array}{r}1.93 \\
(1.19)\end{array}$ & & $2.30(1.44)$ & & $1.24(1.02,1.52)$ & 4.59 (1) & 0.032 \\
\hline Boys* & $\begin{array}{r}0.98 \\
(0.94)\end{array}$ & & $1.20(1.05)$ & & $1.26(0.97,1.63)$ & $3.02(1)$ & 0.082 \\
\hline Girls & $\begin{array}{r}0.95 \\
(0.82)\end{array}$ & & $1.10(1.14)$ & & $1.16(0.90,1.48)$ & $1.30(1)$ & 0.253 \\
\hline $\begin{array}{c}\text { Birth spacing less } \\
\text { than } 2 \text { years } \\
\text { No } \\
\text { Yes }\end{array}$ & & $\begin{array}{l}80(34.6) \\
17(34.0)\end{array}$ & & $\begin{array}{r}151(65.4) \\
33(66.0)\end{array}$ & $1.03(0.54,1.96)$ & $0.01(1)$ & 0.932 \\
\hline $\begin{array}{l}\text { History of mistimed } \\
\text { pregnancy } \\
\text { Yes } \\
\text { No }\end{array}$ & & $\begin{array}{l}23(38.3) \\
74(33.5)\end{array}$ & & $\begin{array}{r}37(61.7) \\
147(66.5)\end{array}$ & $1.24(0.68,2.23)$ & $0.49(1)$ & 0.484 \\
\hline $\begin{array}{c}\text { History of unwanted } \\
\text { pregnancy } \\
\text { Yes } \\
\text { No }\end{array}$ & & $\begin{array}{l}13(37.1) \\
84(34.1)\end{array}$ & & $\begin{array}{r}22(62.9) \\
162(65.9)\end{array}$ & $1.14(0.55,2.38)$ & $0.12(1)$ & 0.727 \\
\hline $\begin{array}{c}\text { History of induced } \\
\text { abortion* } \\
\text { Yes } \\
\text { No }\end{array}$ & & $\begin{array}{r}7(58.3) \\
90(33.5)\end{array}$ & & $\begin{array}{r}5(41.7) \\
179(66.5)\end{array}$ & $2.78(0.86,9.02)$ & $2.92(1)$ & 0.088 \\
\hline $\begin{array}{l}\text { Previous } \\
\text { contraceptive use** }\end{array}$ & & & & & & & \\
\hline No & & $57(76.0)$ & & $18(24.0)$ & 1 & & \\
\hline Yes & & $40(19.4)$ & & $166(80.6)$ & $13.14(6.98,24.74)$ & $63.72(1)$ & $<0.001$ \\
\hline
\end{tabular}




\begin{tabular}{|c|c|c|c|c|c|c|c|}
\hline \multirow{3}{*}{ Factors } & \multicolumn{4}{|c|}{ PPFP use } & \multirow{3}{*}{$\begin{array}{l}\text { Crude odds ratio } \\
\qquad(95 \% \mathrm{Cl})\end{array}$} & \multirow{3}{*}{$\begin{array}{l}\text { Wald sta- } \\
\text { tistics (df) }\end{array}$} & \multirow{3}{*}{$p$-value } \\
\hline & \multicolumn{2}{|c|}{ No $(n=97)$} & \multicolumn{2}{|c|}{ Yes $(n=184)$} & & & \\
\hline & Mean (SD) & n (\%) & Mean (SD) & n (\%) & & & \\
\hline \multicolumn{8}{|l|}{$\begin{array}{l}\text { Contraceptive } \\
\text { health education } \\
\text { Received health } \\
\text { education }\end{array}$} \\
\hline $\begin{array}{r}\text { education } \\
\text { No }\end{array}$ & & $10(40.0)$ & & $15(60.0)$ & 1 & & \\
\hline Yes & & $87(34.0)$ & & $169(66.0)$ & $1.30(0.56,3.00)$ & $0.36(1)$ & 0.547 \\
\hline \multicolumn{8}{|l|}{ Antenatal health } \\
\hline $\begin{array}{l}\text { No } \\
\text { Noucation }\end{array}$ & & $68(37.0)$ & & $116(63.0)$ & 1 & & \\
\hline Yes & & $29(29.9)$ & & $68(70.1)$ & $1.38(0.81,2.33)$ & $1.40(1)$ & 0.237 \\
\hline \multicolumn{8}{|l|}{ In-patient health } \\
\hline $\begin{array}{r}\text { education } \\
\text { No }\end{array}$ & & $38(34.5)$ & & $72(65.5)$ & 1 & & \\
\hline Yes & & $59(34.5)$ & & $112(65.5)$ & $1.00(0.61,1.66)$ & $0.00(1)$ & 0.994 \\
\hline \multicolumn{8}{|l|}{ Postnatal health } \\
\hline $\begin{array}{l}\text { education } \\
\text { No }\end{array}$ & & $72(35.5)$ & & $131(64.5)$ & 1 & & \\
\hline Yes & & $25(32.1)$ & & 53 (67.9) & $1.17(0.67,2.03)$ & $0.29(1)$ & 0.590 \\
\hline \multicolumn{8}{|l|}{$\begin{array}{l}\text { Child immunization } \\
\text { visit health educa- } \\
\text { tion* }\end{array}$} \\
\hline $\begin{array}{l}\text { No } \\
\text { Yes }\end{array}$ & & $\begin{array}{l}53(39.3) \\
44(30.1)\end{array}$ & & $\begin{array}{r}82(60.7) \\
102(69.9)\end{array}$ & $1.50(0.91,2.46)$ & $2.57(1)$ & 0.109 \\
\hline \multicolumn{8}{|l|}{ Individual health } \\
\hline $\begin{array}{l}\text { equcation" } \\
\text { No }\end{array}$ & & $10(55.6)$ & & $8(44.4)$ & 1 & & \\
\hline Yes & & 77 (32.4) & & $161(67.6)$ & $2.61(0.99,6.89)$ & $3.78(1)$ & 0.052 \\
\hline \multicolumn{8}{|l|}{$\begin{array}{l}\text { Spousal involvement } \\
\text { during health edu- }\end{array}$} \\
\hline $\begin{array}{l}\text { cation } \\
\text { Yes } \\
\text { No }\end{array}$ & & $\begin{array}{l}21(36.2) \\
66(33.3)\end{array}$ & & $\begin{array}{r}37(63.8) \\
132(66.7)\end{array}$ & $1.14(0.62,2.09)$ & $0.17(1)$ & 0.685 \\
\hline Knowledge score & $\begin{array}{r}10.54 \\
(3.79)\end{array}$ & & $12.49(3.68)$ & & $1.15(1.07,1.23)$ & $15.69(1)$ & $<0.001$ \\
\hline $\begin{array}{l}\text { Social support } \\
\text { score }\end{array}$ & $\begin{array}{l}24.45 \\
(5.93)\end{array}$ & & $28.30(5.85)$ & & $1.12(1.07,1.17)$ & $23.38(1)$ & $<0.001$ \\
\hline
\end{tabular}

${ }^{*} p$-value $<0.25 \quad{ }^{* *} p$-value $<0.05$

Within the month of delivery, $29.2 \%$ had commenced $(77 \%)$ and intrauterine devices $(71 \%)$ instead of pills PPFP; out of which, $51.7 \%$ had female sterilisation $(65 \%)$ and condoms (51\%).

done together with caesarean delivery, $23.0 \%$ had commenced contraceptive pills, $12.0 \%$ used DMPA Significant factors

injection, $4.8 \%$ had implants inserted while $2.7 \%$ had IUD placed. As $5.8 \%$ of the women had already started The significant factors at univariable analysis with $p$ using condoms within the month of childbirth, this value $<0.05$ were secondary and below level of indicated an early resumption in sexual activity education of women, parity, previous contraceptive following the caesarean delivery. use, knowledge score, and social support score. Employment status, age of husband, household At one year, approximately $65 \%$ of women with recent income, age of marriage, duration of marriage, caesarean delivery had commenced postpartum gravidity, number of boys, history of induced family planning use. Methods that were initiated early abortion, antenatal health education, child health (within 6 months) included implants $(80 \%)$, injections visit health education, and individual health 
education were also included in the multivariable analysis since their $p$-values were $0.05 \leq p<0.25$.

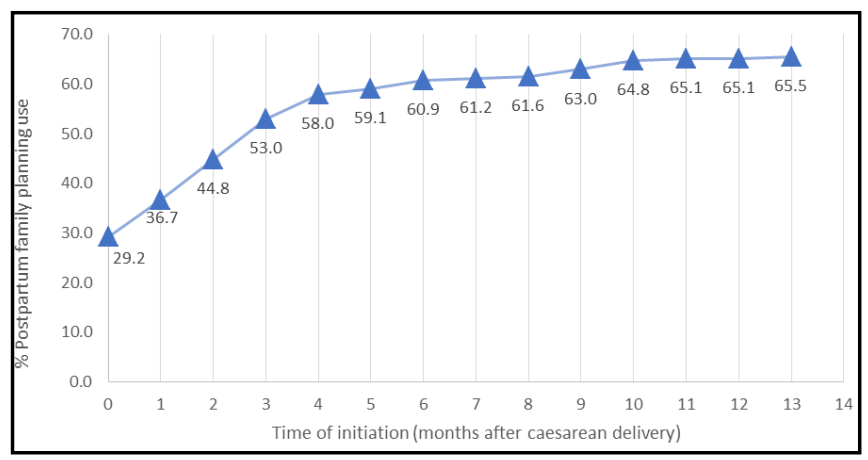

Figure 1: Trend of postpartum family planning initiation after caesarean delivery (by month)

From the multivariable analysis, factors associated with postpartum family planning use included secondary and below level of education of women $(A O R=2.37, \quad 95 \% \quad C l \quad(1.05, \quad 5.34))$ previous contraceptive use $(\mathrm{AOR}=9.82,95 \% \mathrm{Cl}(4.81,20.06))$, individual health education $(\mathrm{AOR}=4.19,95 \% \mathrm{Cl}$ $(1.23,14.30))$, higher knowledge score $(A O R=1.12$, $95 \% \mathrm{Cl}(1.03,1.23))$, and higher social support score $(A O R=1.09,95 \% \mathrm{Cl}(1.03,1.16))$. Women who had a secondary and below level of education were twice as likely to use postpartum family planning than those with tertiary education. Those who had previously used contraceptives were nine times more likely to use postpartum family planning. Women who had been provided with individual health education were four times more likely to use postpartum family planning. Those who had higher knowledge and social support scores had a higher likelihood of using postpartum family panning than those who had lower scores.

\section{DISCUSSION}

PPFP use among women with recent caesarean delivery

We discovered that $65 \%$ of respondents with recent caesarean delivery were using PPFP. This is contrary to findings among postpartum women in general, whereby only one-third of them were using contraceptives. ${ }^{3}$ Women that had caesarean delivery were more likely to use contraceptives following deliveries attended by skilled birth attendants, which had been proven to enhance contraceptive use. $^{23}$ Additionally, fear of subsequent pregnancy may lead women with recent caesarean delivery to use effective contraceptives to prevent pregnancy. ${ }^{23}$ We determined that most respondents were using methods that were highly dependable on "perfect" use, such as pills and condoms.

\section{PPFP initiation}

Early initiation of PPFP is essential, as some women may have their fertility return as early as 6 weeks postpartum ${ }^{24}$, particularly if they were not lactating. Only $30 \%$ had commenced PPFP use within the first month, leaving the remainder at risk if they were not practicing the lactational amenorrhea method (LAM). Furthermore, women that underwent caesarean deliveries were more likely to have no intention to breastfeed, did not initiate breastfeeding, faced breastfeeding difficulties, and more likely to discontinue breastfeeding within the first year. ${ }^{14}$ Nonetheless, LAM only provides protection for up to 6 months following exclusive breastfeeding. As illustrated, early initiation was also significant since only a minor change in uptake was detected after four months following delivery.

\section{Factors of PPFP use}

Although previous research has indicated that women of higher socio-economic status; higher household income ${ }^{17,25,26}$ and higher education level ${ }^{27}$ had a greater likelihood to use contraceptives, our findings indicated higher PPFP use among women with a secondary or lower education level. This indicated that other important determinants influenced PPFP use in our setting, such as previous contraceptive use, individual health education, knowledge, and social support-all of which had significant values in the results. Notably, previous contraceptive use prior to pregnancy significantly 
Table III: Multiple logistic regression analysis of factors associated with PPFP use $(n=281)$

\begin{tabular}{|c|c|c|c|c|c|}
\hline Factors & Adjusted OR (95\% Cl) & $\begin{array}{l}\text { Regression coef- } \\
\text { ficient (B) }\end{array}$ & SE & $\begin{array}{l}\text { Wald stat } \\
\text { (df) }\end{array}$ & $p$-value \\
\hline \multicolumn{6}{|l|}{ Education level of women } \\
\hline Tertiary & 1 & & & & \\
\hline Secondary and below & $2.37(1.05,5.34)$ & 0.86 & 0.42 & $4.32(1)$ & 0.038 \\
\hline \multicolumn{6}{|l|}{ Previous contraceptive use } \\
\hline No & 1 & & & & \\
\hline Yes & $9.82(4.81,20.06)$ & 2.28 & 0.37 & $39.26(1)$ & $<0.001$ \\
\hline \multicolumn{6}{|l|}{ Individual health education } \\
\hline No & 1 & & & & \\
\hline Yes & $4.19(1.23,14.30)$ & 1.43 & 0.63 & $5.24(1)$ & 0.022 \\
\hline Knowledge score & $1.12(1.03,1.23)$ & 0.12 & 0.05 & $6.47(1)$ & 0.011 \\
\hline Social support score & $1.09(1.03,1.16)$ & 0.09 & 0.03 & $9.34(1)$ & 0.002 \\
\hline
\end{tabular}

Constant $=-6.19$

Forward LR method was applied

No multicollinearity and no interaction

Hosmer-Lemeshow test, $\mathrm{p}$-value $=0.733$

Classification table $79.3 \%$ correctly classified

Area under receiver operating

influenced PPFP practice ${ }^{17,26,28}$, as women with prior use had a better knowledge and attitude towards contraceptives. $^{28}$

Although it was hypothesised that knowledge greatly predicts PPFP use, this notion has not been included in most existing studies. Another dilemma is due to an inconclusive association; for example, research conducted in Ethiopia observed a significant association between knowledge and PPFP use ${ }^{25}$, while another study performed in Uganda observed no significant association. ${ }^{26}$ Our findings agree with other studies conducted in Malaysia among women in the general population, whereby knowledge was found to significantly affect contraceptive use. ${ }^{20,29}$ Our study also supports additional findings that indicated a low level of knowledge among women in the general Malaysian population. ${ }^{20,30}$ Despite most of the respondents (91\%) self-declaring as having received contraceptive health education, nearly half of them (43\%) had poor actual knowledge on the topic. Hence, individual health education significantly affects knowledge and PPFP use. As such, individual women would be able to make informed decisions on methods deemed most suitable through developing an awareness and understanding of various contraceptive methods with their respective mode of actions and administration, as well as knowledge on reproductive physiology and pathology.

Notably, husbands, family, and friends served as major sources of influence and information for contraceptive use. ${ }^{25,26}$ Our findings agreed with a previous study conducted among postpartum women in Cambodia, another Asian country, whereby social support was significantly associated with contraceptive use. ${ }^{31}$ Husbands, peers, and elders influenced contraceptive use with the strongest influence being from husbands ${ }^{31}$ since women's compliance to their husband's decisions were considered essential within the culture. ${ }^{32}$

\section{Strengths and limitations}

Although our study was cross-sectional, it provided insight into an Asian country with limited data on postpartum contraceptive use. The study design was acceptable as it allowed us to identify significant factors from the numerous variables included in the study, with adequate sampling being included based on our sample size calculation. Our study provides state-wide data on PPFP use among women with previous caesarean delivery in a setting with the 
lowest contraceptive prevalence in the country using a validated instrument to assess knowledge and social support within the community. Although only women who had caesarean delivery in government tertiary centres were studied, the majority of caesarean sections took place at these centres.

\section{CONCLUSION}

Our study identified modifiable factors that influenced PPFP use, such as individual health education, knowledge, and social support. Additionally, we determined that most contraceptive health education took place within the in-patient setting. Thus, contraceptive counselling should be attempted at each contact with healthcare providers (especially the primary healthcare setting), as women had multiple contacts with these facilities (i.e. during antenatal followups, postnatal home visits, child health and immunisation visits). Moreover, an effort to include husbands during such individualised contraceptive counselling should be undertaken, as previous research has demonstrated that spousal support serves as a crucial component in promoting PPFP practice. Women with multiple risk factors such as advanced maternal age and grand multiparity should be offered a permanent method of contraception or longer-acting reversible methods. Additionally, longer-acting reversible methods should be promoted to enhance postpartum family planning initiation and use.

Future research examining other determinants within the community and policy as well as in-depth assessment of contraceptive service delivery is needed to enhance postpartum family planning services. Identification of these determinants with subsequent strategic planning to augment contraceptive service uptake and compliance would gear Malaysia towards achieving the United Nations Sustainable Development Goals.

\section{CONFLICT OF INTEREST}

None

\section{ACKNOWLEDGEMENTS}

The authors would like to thank the Director General of Health Malaysia for the permission to publish this paper. The authors also would like to thank Dr Fatemeh Najafi-Sharjabad for her permission to utilise the questionnaire for this research. The authors wish to express gratitude to the directors of all the hospitals as well as to the staff involved for their cooperation in this research.

\section{FUNDING}

This research did not receive any specific grant from funding agencies in the public, commercial, or not-for-profit sectors.

\section{REFERENCES}

1. World Health Organization. Programming strategies for Postpartum Family Planning. 2013.

2. World Health Organization. Health in 2015: from MDGs, millennium development goals to SDGs, sustainable development goals. 2015.

3. Gaffield ME, Egan S, Temmerman M. It's about time: WHO and partners release programming strategies for postpartum family planning. Global Health: Science and Practice. 2014;2 (1):4-9.

4. Najimudeen M, Sachchithanantham K. An insight into low contraceptive prevalence in Malaysia and its probable consequences. International Journal of Reproduction, Contraception, Obstetrics and Gynecology. 2017;3(3):493-6.

5. Norbanee THT, Norsa'adah B, Naing NN, Aidawani A, Zainoremi CZ. Choice and discontinuation of contraceptive methods 
among Kelantanese women in Malaysia. International Medical Journal. 2008;15(3):199204.

6. Ab Razak R. Knowledge, attitude and practice of Malay folk methods in family planning. Malaysian journal of reproductive health: a publication of the Reproductive Research Centre of the National Population and Family Development Board, Malaysia. 1985;3(1 Supplement):S64

7. Betrán AP, Ye J, Moller A-B, Zhang J, Gülmezoglu AM, Torloni MR. The Increasing Trend in Caesarean Section Rates: Global, Regional and National estimates: 1990-2014. PloS one. 2016;11(2):1-12.

8. Festin MR, Laopaiboon M, Pattanittum P, Ewens MR, Henderson-Smart DJ, Crowther CA. Caesarean section in four South East Asian countries: reasons for, rates, associated care practices and health outcomes. BMC pregnancy and childbirth. 2009;9(1):17.

9. Bujold E, Gauthier RJ. Risk of uterine rupture associated with an interdelivery interval between 18 and 24 months. Obstetrics \& Gynecology. 2010;115(5):1003-6.

10. Stamilio DM, DeFranco E, Paré E, Odibo AO, Peipert JF, Allsworth JE, et al. Short interpregnancy interval: risk of uterine rupture and complications of vaginal birth after cesarean delivery. Obstetrics \& Gynecology. 2007;110(5):1075-82.

11. World Health Organization. Report of a WHO Technical Consultation on Birth Spacing WHO Press WHO/RHR/071. 2007.

12. Arora M, Jindal S, Gupta S. Retrospective study of cases of rupture uterus. International Journal of Reproduction, Contraception, Obstetrics and Gynecology. 2017;6(5):1900-4.

13. DaVanzo J, Hale L, Razzaque A, Rahman M. The effects of pregnancy spacing on infant and child mortality in Matlab, Bangladesh: how they vary by the type of pregnancy outcome that began the interval. Population studies. 2008;62(2):131-54.

14. Hobbs AJ, Mannion CA, McDonald SW, Brockway M, Tough SC. The impact of caesarean section on breastfeeding initiation, duration and difficulties in the first four months postpartum. BMC pregnancy and childbirth. 2016;16(1):90.

15. Ahmad N, Tey N, Kamarul Zaman K, Muhd Sapri N, Abdul Manaf N, Yeoh Y. Status of family planning in Malaysia. Family Planning in Asia and Pacific Region: National Population and Family Development Board (NPFDB) Bangkok, Thailand; 2010. p. 1-29.

16. Dupont W, Plummer W. Power and Sample Size Calculations for studies Involving Linear Regression, Controlled Clinical Trials. 1998.

17. Jalang'o R, Thuita F, Barasa SO, Njoroge P. Determinants of contraceptive use among postpartum women in a county hospital in rural KENYA. BMC public health. 2017;17(1):604.

18. McLeroy KR, Bibeau D, Steckler A, Glanz K. An ecological perspective on health promotion programs. Health education quarterly. 1988;15 (4):351-77.

19. World Health Organization. Health topics: health education 2018 [29/8/2018]. Available from: http://www.who.int/topics/ health_education/en/.

20. Najafi-Sharjabad F, Hejar Abdul Rahman MH, Yahya SZS. Spousal communication on family planning and perceived social support for contraceptive practices in a sample of Malaysian women. Iranian journal of nursing and midwifery research. 2014;19(7 Suppl1):S19.

21. IBM Corp. IBM SPSS Statistics for Windows. 24.0 ed. Armonk, NY,Released 2016,.

22. Rashed HEM, AWALUDDIN S, Ahmad NA, Supar NHM, Lani ZM, Aziz F, et al. Advanced Maternal Age and Adverse Pregnancy Outcomes in Muar, Johor, Malaysia. Sains Malaysiana. 2016;45 (10):1537-42. 
23. Gebremariam A, Gebremariam H.

Contraceptive use among lactating women in Ganta-Afeshum District, Eastern Tigray, Northern Ethiopia, 2015: a cross sectional study. BMC pregnancy and childbirth. 2017;17 (1):421.

24. Jackson E, Glasier A. Return of ovulation and menses in postpartum nonlactating women: a systematic review. Obstetrics \& Gynecology. 2011;117(3):657-62.

25. Nigussie A, Girma D, Tura G. Postpartum family planning utilization and associated factors among women who gave birth in the past 12 months, Kebribeyah Town, Somali Region, Eastern Ethiopia. J Women's Health Care. 2016;5:340.

26. Sileo KM, Wanyenze RK, Lule H, Kiene SM. Determinants of family planning service uptake and use of contraceptives among postpartum women in rural Uganda. International journal of public health. 2015;60 (8):987-97.

27. Abraha TH, Teferra AS, Gelagay AA. Postpartum modern contraceptive use in northern Ethiopia: prevalence and associated factors. Epidemiology and health. 2017;39.

28. Gebremedhin AY, Kebede Y, Gelagay AA, Habitu YA. Family planning use and its associated factors among women in the extended postpartum period in Addis Ababa, Ethiopia. Contraception and Reproductive Medicine. 2018;3:1.

29. Mansor M, Abdullah KL, Oo SS, Akhtar K, Jusoh AS, Ghazali S, et al. The Prevalence of Family Planning Practice and Associated Factors Among Women in Serdang, Selangor. Malaysian Journal of Public Health Medicine. 2015;15(3):147-56.

30. Shafei M, Shah M, Tengku Ismail T. Knowledge and attitude towards family planning practice and prevalence of short birth spacing among residents of suburban area in Terengganu,
Malaysia. J Community Med Health Educ. 2012;2(180):2161-0711.1000180.

31. Samandari G, Speizer IS, O'Connell K. The role of social support and parity on contraceptive use in Cambodia. International perspectives on sexual and reproductive health. 2010:12231.

32. Wong LP. An exploration of knowledge, attitudes and behaviours of young multiethnic Muslim-majority society in Malaysia in relation to reproductive and premarital sexual practices. BMC public health. 2012;12(1):865. 